\title{
Analysis of spatial cross-correlations in multi-constituent volume data
}

\author{
Journal of Microscopy vol. 232 (2) (2008)
}

\author{
A. Rack ${ }^{1,2, *}$ L. Helfen ${ }^{2}$, T. Baumbach ${ }^{2}$, S. Kirste ${ }^{1}$, \\ J. Banhart ${ }^{1,5}$, K. Schladitz ${ }^{3}$, J. Ohser ${ }^{4}$
}

August 5, 2008

\begin{abstract}
We investigate spatial cross-correlations between two constituents, both belonging to the same microstructure. These investigations are based on two approaches: one via the measurement of the cross-correlation function while the second uses the spatial distances between the constituents. The cross-correlation function can be measured using the fast Fourier transform, while the the distances are determined via the Euclidean distance transform. The characteristics are derived from volume images obtained by synchrotron microtomography. As an example we consider pore formation in metallic foams, knowledge of which is important to control the foam production process. For this example we discuss the spatial crosscorrelation between the pore space and the blowing agent particles in detail.
\end{abstract}

Keywords: microtomography, image analysis, random sets, Euclidean distance transform, Fourier transform, microstructure of foam, CT

\section{Introduction}

In recent years sophisticated imaging methods have been established which deliver high quality volume data. Due to the use of various contrast modes the identification of different constituents (or material phases) inside these images is possible, even with spatial resolutions allowing to depict features down to the submicrometer range. In particular non-destructive three-dimensional (3D) $\mathrm{X}$-ray imaging methods have been used heavily in material science within the past years to investigate fibre structures, sinter processes, crack formation in rocks, metal matrix composites, metallic foams or syntactic foams.

\footnotetext{
${ }^{*}$ Correspondence to: A. Rack, Forschungszentrum Karlsruhe, Institute for Synchrotron Radiation - ANKA, Postfach 3640, D-76021 Karlsruhe, Germany, E-mail: arack@snafu.de

${ }^{1}$ Hahn-Meitner Institute / Helmholtz Centre Berlin, Department Materials, Germany

${ }^{2}$ Institute for Synchrotron Radiation, Forschungszentrum Karlsruhe - ANKA, Germany

${ }^{3}$ Fraunhofer-Institut für Techno- und Wirtschaftsmathematik, Fraunhofer-Platz 1, 67663 Kaiserslautern, Germany, katja.schladitz@itwm.fraunhofer.de

${ }^{4}$ Hochschule Darmstadt, Schöfferstraße 3, D-64295 Darmstadt, jo@h-da.de

${ }^{5}$ Technical University Berlin, Institute of Materials Science and Technology, Germany
} 
The increasing number of investigations using multi-dimensional imaging techniques leads to a high demand for image analysis methods for deriving quantitative results from the measurements. Amongst others methods are needed for finding dependencies between the positions of different constituents within the image. That is, to decide whether correlations exist and of what type they are.

In signal processing analyzing correlations between two signals - called crosscorrelations - is a standard task, usually solved with the help of Fourier methods. Spatial cross-correlation can be captured mathematically by the crosscorrelation measure for any two arbitrary random measures associated with random structures based on the same probability space. A fundamental theoretical introduction to cross-correlation measures is given in Stoyan \& Ohser (1984); Lhotský (2006). Cross-correlations in marked point processes have been studied in Stoyan (1984b,a).

For ecological data, the spatial cross-correlations between a random point field, a random fiber system, and random sets were studied in Stoyan \& Ohser (1982), where the points are sites of trees, the fibers are river courses, and the random sets are regions of specific soil types. Between such structures there may exist mutual relationships, e. g. attraction between the point sites and the river courses or inhibition between sites and soil types. A further application in ecology is considered in Reich et al. (1994). For applications in geoscience see e.g. Agterberg \& Fabbri (1979); Duffy \& Hughes-Clarke (2005), and for an application in materials science see Jeulin (1986). In signal processing, spatial cross-correlation of multi-antenna systems is considered in Jorswieck \& Sezgin (2004). In Section 3.1 we consider the cross-correlation of the volume measures associated with two constituents.

A first approach to investigate correlations in metallic foams using morphological transformations is reported in Helfen et al. (2003, 2005). This method was applied successfully to various cases. However, the enormous computational effort and the coarse grid of analyzable distance values motivates a refinement resulting in the method presented in Section 3.3, where we investigate the distances between two constituents. More precisely, consider a point randomly chosen in the first constituent. The smallest distance to the second constituent is a random variable and its distribution can provide information about the stochastic dependence or independence of the two constituents. This kind of information is different from the information contained in cross-correlation functions. Distance methods in the investigation of stochastic dependence (or stochastic independence) were first used for marked point fields in Ma et al. (2006). In Section 3.3 we introduce a distance method characterizing stochastic dependence of two random sets.

We demonstrate the two methods for detecting spatial correlations between different microstructural constituents using the example of metal foams. Due to their high specific stiffness and low density, metal foams have a large potential for industrial applications in all kinds of light-weight constructions (Banhart \& Weaire, 2002). Various production techniques are known to produce metal foams (Banhart, 2001), a common one is the powder-metallurgical route ( $\mathrm{Yu}$ et al., 1998). Here the metal or alloy to be foamed in powder form is mixed with a blowing agent and then compacted in order to create a solid precursor material. The precursor is heated in a furnace. Ideally, the blowing agent starts to release gas which then forms the pores at the same temperature at which 


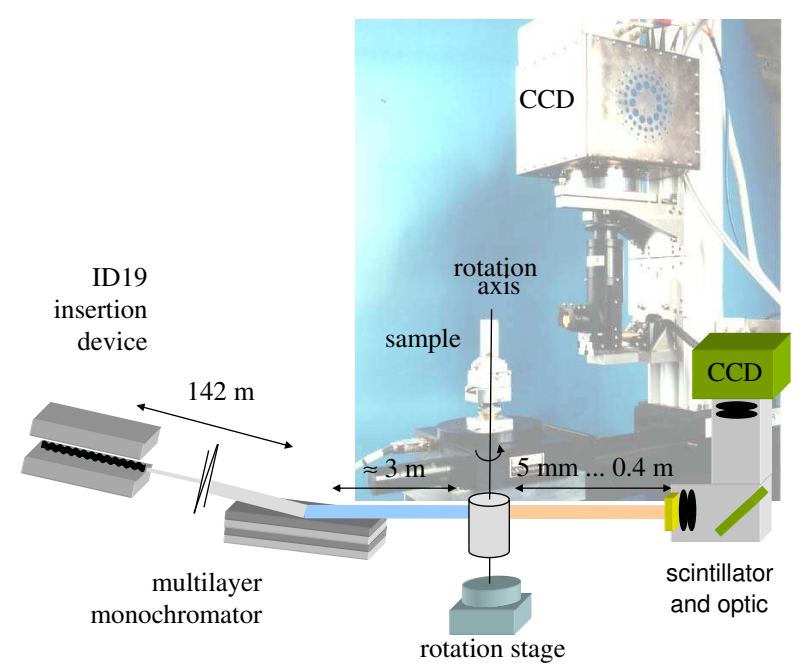

Figure 1: Sketch of the synchrotron microtomography facility at the beamline ID19 of the ESRF with embedded photo of the tomographic imaging station in the background (Cloetens et al., 1999).

the alloy transforms from solid into a mushy state. Quenching of the sample at the desired expansion state conserves the pore structure, resulting in a metallic foam.

One focus of research on metallic foams lies on pore formation, as an understanding of this process promises to help to control the final pore structure. The powder mixing process itself is stochastic and so far yields no possibility to influence the resulting foam. However, only a reproducible homogeneous pore structure guarantees the properties and quality of a metallic foam and thus opens the market for mass production.

We analyse metallic foam samples made of commercial AW-6061 and AlSi7 with $\mathrm{TiH}_{2}$ as blowing agent in early foaming stages. As imaging method, synchrotron microtomography is chosen since it is non-destructive, yields a good material contrast and high spatial resolutions (Flannery et al., 1987). Experiments are carried out at the ID19 of the European synchrotron ESRF. The key question to be answered when investigating these samples is whether two different types of pores - called type-I and type-II - do exist. Type-I pores are inflated in the vicinity of the blowing agent while type-II pores form at sites spatially independent of the blowing agent (Bellmann et al., 2001). The existence of type-II pores would indicate that the position of early pores in metallic foams can be influenced and thus lead to means for pore structure controling. In order to answer that question we investigate whether the pore space and the positions of the $\mathrm{TiH}_{2}$ particles are spatially dependent in the two foam samples.

\section{Microtomography}

The acronym tomography nowadays summarizes imaging methods which deliver $3 \mathrm{D}$ data sets consisting of cross-sectional slices from the investigated sample ei- 
ther by non-destructive imaging (e.g. using X-rays as in our case) or destructive analysis (e.g. position sensitive ion microscopy - 3D atom probe). Godfrey N. Hounsfield introduced the first apparatus to examine non-destructively crosssections of a body in the 1970's, based on an idea which had been published already by Allan C. Cormack in the 1960's. For 3D computed tomography, the radiographic image interpreted as projection of the linear X-ray attenuation coefficient can be used to reconstruct the mass distribution within a sample by combining the projection data from a set of different projection angles. This method frequently called computed tomography (CT) had a strong impact to medicine, see Hsieh (2003), and was introduced soon in materials science as well (Reimers \& Goebbels, 1983). Meanwhile, resolution has been pushed down to the micrometer-scale (microtomography - $\mu \mathrm{CT}$ (Flannery et al., 1987)), presently limited by the flux and spot size of the microfocus X-ray tubes, cf. Banhart (2007).

In order to overcome these limitations, synchrotron radiation can be applied (Flannery et al., 1987; Bonse \& Busch, 1996), in particular when high sensitivity, fast data acquisition, and high spatial resolution are required. Here, the available flux is orders of magnitudes higher than compared to X-ray tubes, delivering radiographic images with a low noise level while requiring exposure times in the (sub-)second range only. The high flux also allows to monochromatise the radiation leading to a higher contrast within the images. Moreover, the nearly parallel beam at typical synchrotron imaging setups increases image quality as the reconstruction can be performed slice by slice, e. g. by eliminating so-called cone beam reconstruction artefacts. These artefacts appear when using a non-parallel X-ray beam for imaging due to the overlap of projection information from different imaged cross-sections of the specimen.

A typical setup for synchrotron microtomography $(\mathrm{S} \mu \mathrm{CT})$ without using focusing X-ray optics is displayed in Figure 1. The synchrotron radiation is monochromatised, e.g. by using multilayer mirrors, and the sample is placed at an appropriately large distance from the source in order to eliminate the influence of the finite source size. Besides the high-precision sample manipulator (for the alignment of the rotation axis and to perform a scan with low sample run-out), the important device for microtomography is a detector-microscope designed to obtain highly resolved radiographic projection images, here using the concept of Bonse \& Busch (1996): a scintillator screen (powder or single crystal) converts the X-ray photons of the transmitted beam into visible light photons. The microscope optics behind images the luminescent screen onto a CCD chip. The magnification factor here is given by the ratio of the objective's and the tube lens's focus lengths. In the parallel section of the optical path a diaphragm can be placed to optimise the ratio of absorbed X-ray photons (scintillator) vs. detected luminescence photons (CCD) and to adapt the numerical aperture to the resolution used. The maximal lateral resolution is determined by the microscopes' optics, the scintillating screen, the CCD chip, and the sampling theorem, and can extend down to the submicrometer range (Banhart, 2007).

Tomographic techniques are applied in order to determine the spatial distribution of constituents represented. Such a spatial distribution is usually represented by a random function $f: R^{3} \mapsto \mathbb{R}^{+}$reconstructed from a set of two-dimensional projection images, where $\mathbb{R}^{3}$ is the Euclidean space and $\mathbb{R}^{+}$ denotes the positive half-axes. For the reconstruction of the tomographic images the filtered backprojection algorithm is frequently used, see e. g. Banhart 

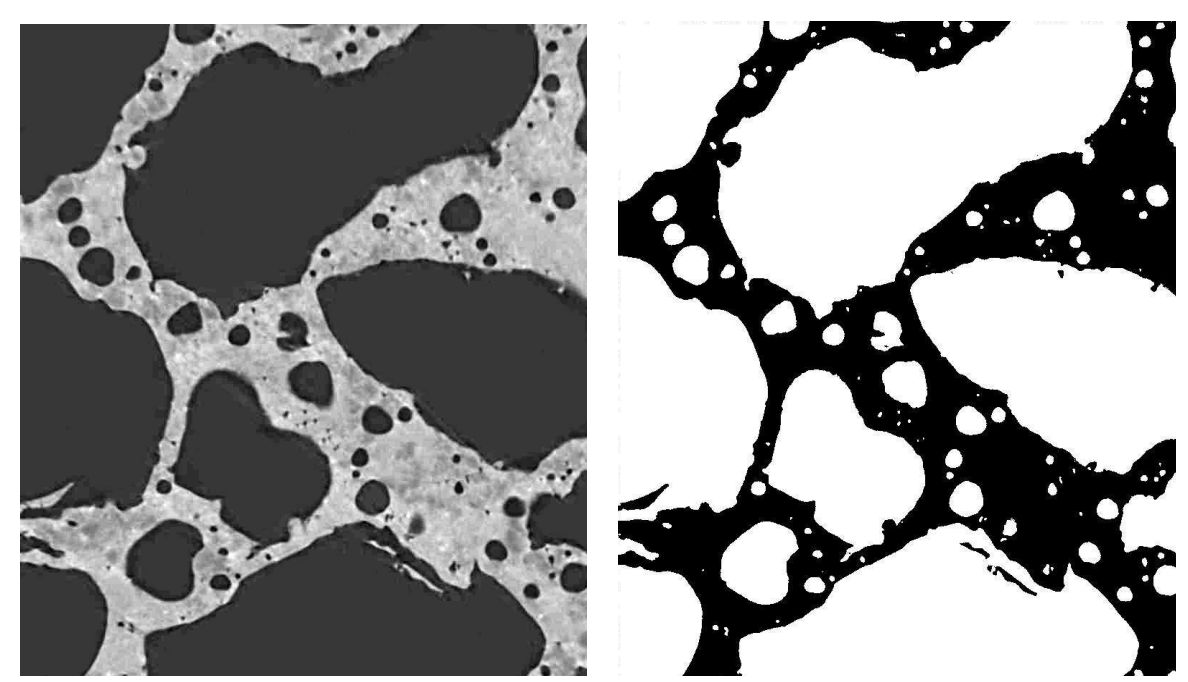

Figure 2: Tomographic slice of an AlSiCu metallic foam (left) and corresponding Boolean image (right) containing only the morphological pore information (Rack, 2006).

(2007). For classical absorption $\mu \mathrm{CT}$, the function $f$ corresponds to the linear $\mathrm{X}$-ray attenuation coefficient.

With the ongoing development, more sophisticated tomographic methods have been introduced, allowing to use other contrast modes with higher sensitivity such as the local electron density (holotomography), the chemical species distribution (fluorescence tomography), the inner surfaces and interfaces (refraction enhanced tomography), or the local crystalline lattice quality (topotomography) - for further details see e. g. Banhart (2007).

Synchrotron tomography as modern, non-destructive and three-dimensional microscopy can deliver volume images with a very good signal-to-noise ratio and a high contrast between different material phases: ideal conditions to apply a subsequent 3D image analysis. Nevertheless, one has to keep in mind that, compared to established laboratory methods, synchrotron radiation is expensive and available beamtime is limited, which restricts its application to selected investigations.

\section{Image processing and analysis}

In general, tomographic data sets are volume images in the sense of a 3D-matrix filled with (e.g. grey-scaled) values. Depending on the acquisition method and contrast mode these values can represent, e.g., hydrogen concentration (Magnetic resonance imaging), density of metabolized isotopes (Positron emission tomography), the electron density (holotomography), or as in our case the linear X-ray attenuation coefficient $(\mu \mathrm{CT})$.

The first step of our image analysis is to identify and subsequently separate in the data sets the different constituents. Each of the constituents is represented as a Boolean image (also called binary image) allowing the application of further data processing exclusively on this component. We use the difference between 
the (grey) values as contrast criterion. Various techniques for separation have been developed (Pal \& Pal, 1993; Pal \& Bhandari, 1993; Wirjadi, 2007), ranging from simple global thresholding to complex stochastic algorithms. We chose a region-growing algorithm in combination with a threshold hysteresis as this is known to be robust with respect to noise, relatively easy to handle and to reduce segmentation artefacts. A typical result can be seen in Figure 2. The thresholds were chosen manually.

We now introduce two algorithms for investigating spatial dependence in multi-constituent tomographic images. The capital letters $\Xi, \Psi, \ldots$ denote random closed sets in $\mathbb{R}^{3}$ associated with constituents of macroscopically homogeneous microstructures. Here macroscopic homogeneity means that the distribution of each constituent is invariant with respect to all translations. (Notice that in Stochastic Geometry a macroscopically homogeneous constituent is said to be stationary.) Furthermore, the realizations of the random sets are assumed to belong to the extended convex ring or to be topological closures of the complement of elements of the extended convex ring. The sets $\Xi, \Psi, \ldots$ are supposed to be jointly simultaneously macroscopically homogeneous, i. e. the distribution of $(\Xi+x, \Psi+x, \ldots)$ is invariant with respect to all shifts $x \in \mathbb{R}^{3}$ as well.

\subsection{Spatial cross-correlation}

It is well known that second order characteristics like cross-correlations as well as their counterparts in frequency space can be measured from images of the microstructures, see Frank (1980); Manolakis \& Proakis (1996). The fast Fourier transform (FFT) and sophisticated algorithms for its computation (Marcotte, 1996) allow to determine these quantities fast and efficiently. The idea to measure second order characteristics in real space via the Fourier transform has been around for some time (Torquato (2002), see also Ohser \& Mücklich (2000), Chapter 5), too. A sound mathematical basis for the computation of the autocorrelation function of a materials constituent and its counterpart in frequency space - the so-called Bartlett spectrum of the constituent - is provided in Koch et al. (2003); Ohser et al. (2005).

In this section we consider the cross-correlation of the volume measures associated with two random sets. Several problems arise in their computation via frequency space and are addressed in the following. First, it should be noted that the Fourier transform of a random set does not exist, i.e. the indicator function of the set representing this constituent is not Fourier transformable. Second, there is no spectral measure (a Bartlett spectrum) associated with the cross-correlation function, since the cross-correlation function in general is not positive definite. Finally, the fast Fourier transform (FFT) supposes periodicity with respect to the window $W$ through which the microstructure is observed. However, the microstructure is not periodic (in particular not $W$-periodic). In higher dimensions the fraction of $W$ close to the boundary is larger than in the one-dimensional case. Thus, the windowing successfully applied in onedimensional cases can lead to a considerable bias in higher dimensions.

Consider now two macroscopically homogeneous random sets $\Xi, \Psi$ in $\mathbb{R}^{3}$. Let $V(\Xi \cap \cdot)$ and $V(\Psi \cap \cdot)$ denote the random volume measures of $\Xi$ and $\Psi$, respectively, and let $M^{(2)}$ be the mixed second order moment measure defined 
as expectation $\mathbb{E}$ of the product of the volume measures,

$$
M^{(2)}(A \times B)=\mathbb{E}(V(\Xi \cap A) V(\Psi \cap B))
$$

for any compact sets $A, B \subset \mathbb{R}^{3}$. From the joint macroscopic homogeneity of $\Xi$ and $\Psi$ it follows that $M^{(2)}$ is invariant with respect to diagonal shifts of $A \times B$, i. e.

$$
M^{(2)}(A \times B)=M^{(2)}((A+x) \times(B+y))
$$

for all translations of $A \times B$ in $\mathbb{R}^{6}$ along the diagonal $x=y$. Then the probability $\mathbb{P}(x \in \Xi, y \in \Psi)$ depends on the difference $h=y-x$ only. The function $\operatorname{cov} \Xi, \Psi: \mathbb{R}^{n} \mapsto \mathbb{R}$ defined by

$$
\begin{aligned}
\operatorname{cov} \Xi, \Psi & =\mathbb{P}(x \in \Xi, x+h \in \Psi)-\mathbb{P}(x \in \Xi) \mathbb{P}(x+h \in \Psi) \\
& =\mathbb{E}\left(\left(\mathbf{1}_{\Xi}(0)-V_{V}(\Xi)\right)\left(\mathbf{1}_{\Psi}(h)-V_{V}(\Psi)\right)\right)
\end{aligned}
$$

is called the cross-covariance function of the random sets $\Xi$ and $\Psi$. Here $\mathbf{1}$ is the indicator, $V_{V}(\Xi)$ and $V_{V}(\Psi)$ denote the volume densities of $\Xi$ and $\Phi$, respectively,

$$
V_{V}(\Xi)=\mathbb{E} \mathbf{1}_{\Xi}(x), \quad V_{V}(\Psi)=\mathbb{E} \mathbf{1}_{\Psi}(x), \quad x \in \mathbb{R}^{3} .
$$

The cross-correlation function $\operatorname{cor}_{\Xi, \Psi}$ of the random sets $\Xi$ and $\Psi$ is the normalised cross-covariance function. For positive volume densities

$$
\operatorname{cor}_{\Xi, \Psi}(x)=\frac{\operatorname{cov} \Xi, \Psi_{\Xi}(x)}{V_{V}(\Xi) V_{V}(\Psi)}, \quad x \in \mathbb{R}^{3} .
$$

For the sake of easily readable formulae we consider just the cross-covariance function in the following examples. The deduced properties carry over to the cross-correlation function in a straightforward manner.

\section{Examples}

(i) Clearly, for independent random sets $\Xi, \Psi$ it follows that $\operatorname{cov} \Xi, \Psi(x)=0$ for all $x \in \mathbb{R}^{3}$. However, the condition that the cross-covariance vanishes is necessary but not sufficient for the independence of $\Xi$ and $\Psi$.

(ii) Let $\Xi$ be a macroscopically homogeneous random closed set with a volume density $0<V_{V}(\Xi)<1$. If $\Psi$ is the topological closure $\overline{\Xi^{c}}$ of the complementary set $\Xi^{c}$ of $\Xi$. Then $\Psi=\overline{\Xi^{c}}$ inherits the macroscopic homogeneity of $\Xi$ and $V_{V}(\Psi)=1-V_{V}(\Xi)$. Moreover, the sets $\Xi$ and $\Psi$ are dependent random variables.

Note that for most constituents of materials structures the cross-covariance

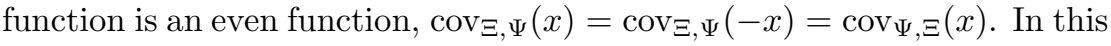
case, the identity

$$
\begin{aligned}
\mathbb{P}(x \in \Xi, y \in \Xi)+\mathbb{P}(x \in \Xi, y \in \Psi) \\
\quad+\mathbb{P}(x \in \Psi, y \in \Xi)+\mathbb{P}(x \in \Psi, y \in \Psi)=1, \quad x, y \in \mathbb{R}^{3},
\end{aligned}
$$

yields

$$
\operatorname{cov}_{\Xi, \Psi}(x)=1-\frac{1}{2}\left(\operatorname{cov}_{\Xi, \Xi}(x)-\operatorname{cov}_{\Psi, \Psi}(x)\right), \quad x \in \mathbb{R}^{3},
$$


where $\operatorname{cov}_{\Xi, \Xi}$ and $\operatorname{cov}_{\Psi, \Psi}$ are the auto-covariance functions of the foreground set $\Xi$ and the background set $\Psi$, respectively. Hence, independence can not be expected as the auto-covariance differs from zero in general. That means, foreground (which may represent a material's constituent) and background (the matrix) are correlated in general.

(iii) Given two random closed sets $\Xi$ and $\Psi, 0<V_{V}(\Xi)<1$ and $V_{V}(\Psi)>$ 0 , we introduce a further random set $\Psi^{\prime}$ which is the intersection of $\Psi$ and the topological closure of the complementary set of $\Xi$, i.e. $\Psi^{\prime}=$ $\Psi \cap \overline{\Xi^{c}}$. The sets $\Xi$ and $\Psi^{\prime}$ depend on each other even if $\Xi$ and $\Psi$ are independent random sets. Assume now that $\Psi$ is not observable directly, i. e. 'information' on $\Psi$ can be gained by observing $\Xi$ and $\Psi^{\prime}$ only. If $\Xi$ and $\Psi$ are independent random sets, then $\Psi$ is also independent of $\overline{\Xi^{c}}$ and for all $x, y \in \mathbb{R}^{3}$

$$
\begin{aligned}
\mathbb{P}\left(x \in \Xi, y \in \Psi^{\prime}\right) & =\mathbb{P}\left(x \in \Xi, y \in \overline{\Xi^{c}}, y \in \Psi\right) \\
& =\mathbb{P}\left(x \in \Xi, y \in \bar{\Xi}^{c}\right) \mathbb{P}(y \in \Psi) .
\end{aligned}
$$

In terms of the cross-covariance functions $\operatorname{cov}_{\Xi, \Psi^{\prime}}$ and $\operatorname{cov}_{\Xi, \overline{\Xi^{c}}}$ the above identity is equivalent to

$$
t_{1}(x)=\frac{\operatorname{cov} \Xi, \Psi^{\prime}}{(x)}=1
$$

for all $x$ with $\operatorname{cov}_{\Xi \Xi \overline{\Xi c}}(x) \neq 0$. The relationship $t_{1}(x) \equiv 1$ serves as a necessary (but not sufficient) criterion for the sets $\Xi$ and $\Psi$ to be independent.

\subsection{Estimation of the cross-correlation function}

Denote by $\mathbb{C}$ the space of complex numbers. In the continuous case the Fourier transform $\hat{f}=\mathscr{F} f$ of a measurable function $f: \mathbb{R}^{3} \mapsto \mathbb{C}$ is defined by

$$
\mathscr{F}[f](\xi)=\frac{1}{(2 \pi)^{3 / 2}} \int_{\mathbb{R}^{3}} f(x) \mathrm{e}^{-i \xi x} d x, \quad \xi \in \mathbb{R}^{3} .
$$

Notice that the function $\hat{f}$ is usually complex-valued, $\hat{f}: \mathbb{R}^{3} \mapsto \mathbb{C}$, even in the case when $f$ is only real-valued. Analogously, the Fourier cotransform or the inverse Fourier transform $\check{f}=\overline{\mathscr{F}} f$ of $f$ is defined by

$$
\overline{\mathscr{F}}[f](x)=\frac{1}{(2 \pi)^{3 / 2}} \int_{\mathbb{R}^{3}} f(\xi) \mathrm{e}^{i x \xi} d \xi, \quad x \in \mathbb{R}^{3} .
$$

The Fourier transform and cotransform are linear and for any integrable function $f$, the inversion formulae $\overline{\mathscr{F}} \mathscr{F} f=f$ and $\mathscr{F} \overline{\mathscr{F}} f=f$ are valid.

The Fourier method is now applied to estimate the spatial cross-correlation function. To this end, we consider again the cross-covariance function since an estimator for this function immediately yields a ratio unbiased estimator for the cross-correlation function.

First we remark that the cross-covariance function is continuous but in general not positive definite. Hence, Bochner's theorem can not be applied in order to introduce a spectral measure associated with the cross-covariance function. 
Until now it is not known if there is any counterpart of the cross-covariance function in inverse space at all.

We consider the case where $\Xi$ and $\Psi$ are observed through the same window $W$. Let $W$ be compact with nonempty interior and containing the origin. In order to give an estimator of the cross-covariance function of two macroscopically homogeneous random closed sets $\Xi$ and $\Psi$, we introduce the windowed functions

$f_{W}(x)=\mathbf{1}_{W}(x)\left(\mathbf{1}_{\Xi}(x)-V_{V}(\Xi)\right), \quad g_{W}(x)=\mathbf{1}_{W}(x)\left(\mathbf{1}_{\Psi}(x)-V_{V}(\Psi)\right), \quad x \in \mathbb{R}^{3}$, associated with $\Xi$ and $\Psi$, respectively. The boundedness of $W$ ensures the integrability of $f_{W}$ and $g_{W}$, and hence, their Fourier transforms $\hat{f}_{W}$ and $\hat{g}_{W}$ exist almost surely. Furthermore, let $c_{W}=\mathbf{1}_{W} * \mathbf{1}_{\breve{W}}$ denote the window function of $W$, where $*$ is the convolution and $\breve{W}$ is the reflection of $W$ at the origin. Furthermore, let $\overline{f(x)}$ denote the complex conjugate of $f(x)$. Then, analogously to the estimator of the auto-covariance function of a random set $\Xi$ described in Koch et al. (2003) and Ohser et al. (2005), the cross-covariance function of $\Xi$ and $\Psi$ can be estimated via

$$
\frac{(2 \pi)^{3 / 2} \overline{\mathscr{F}}\left(\left|\overline{\hat{f}}_{W} \hat{g}_{W}\right|\right)(x)}{c_{W}(x)}
$$

for all $x$ belonging to the interior of $W$. The estimator given above is unbiased for $\operatorname{cov} \Xi, \Psi$.

To our image data given on lattices we apply a discrete version of the Fourier transform, which allows the estimation of the cross-covariance function (and thus of the cross-correlation function too) with a complexity of $\mathcal{O}(n \log n)$, where $n$ is the number of pixels in the Boolean images corresponding to $\Xi$ and $\Psi$, respectively.

\subsection{Distance method}

In this section we describe an alternative approach for characterizing stochastic dependence of two random sets using the distances between them. More precisely, we consider the distribution of the smallest distance from a point in the first set to the second set and exploit the information about the stochastic dependence of the two sets it yields.

Similar to the fast Fourier transform for cross-correlation, the Euclidean distance transform (EDT) is a tool which allows fast computation of quantities like the spherical contact distribution function characterizing shortest distances between random sets, see Mayer (2004). The EDT can be computed in linear time, or very efficiently with quasi-linear behaviour (Cuisenaire, 1999, Chapter $5)$.

Let $\|x-y\|$ denote the Euclidean distance of two points $x, y \in \mathbb{R}^{3}$. The shortest distance

$$
\operatorname{dist}(x, \Xi)=\inf \{\|x-y\|: y \in \Xi\}
$$

between a given point $x \in \mathbb{R}^{3}$ and the random set $\Xi$ is a random variable and the mapping $\operatorname{dist}(\cdot, \Xi)$ can be considered as a random field. If $\Xi$ is macroscopically homogeneous then $\operatorname{dist}(\cdot, \Xi)$ too, i. e. the distribution of dist $(x, \Xi)$ is independent of the position $x$.

We consider the probability that the distance $\operatorname{dist}(x, \Xi)$ is less than a given value $r \geq 0$ under the condition that $x$ belongs to $\Psi$. Let $A \oplus B=\{x+y$ : 
$x \in A, y \in B\}$ denote the Minkowski addition of the sets $A, B \subset \mathbb{R}^{3}$. For the particular case of a ball $B_{r}$ with radius $r$ and centred at the origin it follows that $A \oplus B_{r}=\left\{x \in \mathbb{R}^{3}: \operatorname{dist}(x, A) \leq r\right\}$. If $\mathbb{P}(x \in \Psi)>0$ then

$$
\begin{aligned}
\mathbb{P}(\operatorname{dist}(x, \Xi) \leq r \mid x \in \Psi) & =\frac{\mathbb{P}(\operatorname{dist}(x, \Xi) \leq r, x \in \Psi)}{\mathbb{P}(x \in \Psi)} \\
& =\frac{\mathbb{P}\left(x \in\left(\Xi \oplus B_{r}\right) \cap \Psi\right)}{\mathbb{P}(x \in \Psi)}
\end{aligned}
$$

for all $x \in \mathbb{R}^{3}$ and $r>0$. The conditional probability considered above is independent of $x$ and it follows that

$$
\begin{aligned}
& \mathbb{P}(\operatorname{dist}(x, \Xi) \leq r \mid x \in \Psi) \\
& \quad=\frac{V_{V}\left(\left\{x \in \mathbb{R}^{3}: \operatorname{dist}(x, \Xi) \leq r\right\} \cap \Psi\right)}{V_{V}(\Psi)}, \quad r \geq 0 \\
& \quad=\frac{V_{V}\left(\left(\Xi \oplus B_{r}\right) \cap \Psi\right)}{V_{V}(\Psi)}, \quad r \geq 0 .
\end{aligned}
$$

In the following we write $F_{\Xi, \Psi}(r)=\mathbb{P}(\operatorname{dist}(x, \Xi) \leq r \mid x \in \Psi)$ for short. Notice that $F_{\Xi, \Psi}$ can be considered as a probability distribution function.

\section{Examples}

(i) If $\Xi$ and $\Psi$ are independent random sets, we obtain

$$
F_{\Xi, \Psi}(r)=\mathbb{P}(\operatorname{dist}(x, \Xi) \leq r)=V_{V}\left(\Xi \oplus B_{r}\right), \quad r \geq 0 .
$$

That is, stochastic independence of $\Xi$ and $\Psi$ implies that the function $F_{\Xi, \Psi}$ is independent of $\Psi$.

(ii) Let now $0<V_{V}(\Xi)<1$ and set $\Psi=\Xi^{c}$. From the formulae derived above, the macroscopic homogeneity of $\Xi$, and the fact that the edge of $\bar{\Xi}^{c}$ is almost surely a set of measure 0 , it follows that

$$
\begin{aligned}
F_{\Xi, \overline{\Xi^{c}}}(r) & =\mathbb{P}\left(\operatorname{dist}(x, \Xi) \leq r \mid x \in \overline{\Xi^{c}}\right) \\
& =\frac{V_{V}\left(\left(\Xi \oplus B_{r}\right) \cap \Xi^{c}\right)}{V_{V}\left(\Xi^{c}\right)} \\
& =\frac{V_{V}\left(\Xi \oplus B_{r}\right)-V_{V}(\Xi)}{1-V_{V}(\Xi)}, \quad r \geq 0 .
\end{aligned}
$$

The function $F_{\Xi, \overline{\Xi^{c}}}$ is the spherical contact distribution function of $\Xi$ well known in stochastic geometry.

(iii) Let $\Xi$ and $\Psi$ be independent macroscopically homogeneous random sets with $0<V_{V}(\Xi)<1$ and $V_{V}(\Psi)>0$. As in Example (iii) on Page 8 we define $\Psi^{\prime}=\Psi \cap \overline{\Xi^{c}}$. Then it follows that

$$
\begin{aligned}
F_{\Xi, \Psi^{\prime}}(r) & =\mathbb{P}\left(\operatorname{dist}(x, \Xi) \leq r \mid x \in \Psi^{\prime}\right) \\
& =\frac{\mathbb{P}\left(\operatorname{dist}(x, \Xi) \leq r, x \in \Psi, x \in \overline{\Xi^{c}}\right)}{\mathbb{P}\left(x \in \Psi, x \in \overline{\Xi^{c}}\right)} \\
& =\frac{\mathbb{P}\left(\operatorname{dist}(x, \Xi) \leq r, x \in \overline{\Xi^{c}}\right)}{\mathbb{P}\left(x \in \overline{\Xi^{c}}\right)} \\
& =F_{\Xi, \overline{\Xi^{c}}}(r), \quad r>0 .
\end{aligned}
$$


As a consequence, the ratio $t_{2}$ defined by

$$
t_{2}(r)=\frac{F_{\Xi, \Psi^{\prime}}(r)}{F_{\Xi, \Xi^{c}}(r)}, \quad r \geq 0
$$

can be used to check independence of $\Xi$ and $\Psi$. Stochastic dependence of $\Xi$ and $\Psi$ can be assumed if $t_{2}$ differs from 1 .

The function $F_{\Xi, \Psi}$ can be computed based on (2) or (3), respectively. We apply (2) as this leads to a more efficient method.

Assume that both $\Xi$ and $\Psi$ are observed through a compact window with nonempty interior. The distance dist $(\cdot, \Xi)$ is closely related to the Euclidean distance transform $\mathrm{EDT}_{\Xi^{c}}$ of $\Xi^{c}$ which maps to each point of $\mathbb{R}^{3}$ its shorted distance to $\Xi$,

$$
\operatorname{EDT}_{\Xi^{c}}: \mathbb{R}^{3} \mapsto[0, \infty): x \mapsto \operatorname{dist}(x, \Xi) .
$$

Now the Euclidean distance transform is masked with the random set $\Psi$ as well as with the reduced window $W \ominus B_{r}$ where $\ominus$ denotes the Minkowski subtraction defined by $A \ominus B=\left(A^{c} \oplus B\right)^{c}$ for sets $A, B \subseteq \mathbb{R}^{3}$. This means we consider the product

$$
\mathrm{EDT}_{\Xi^{c}} \mathbf{1}_{\Psi} \mathbf{1}_{W \ominus B_{r}} .
$$

The observation of distances less than $r$ in the reduced window is free of edge effects. Hence, for known volume density $V_{V}(\Psi)$ the ratio

$$
\frac{\operatorname{vol}\left(\left\{x \in \mathbb{R}^{3}:\left(\mathrm{EDT}_{\Xi^{c}} \mathbf{1}_{\Psi} \mathbf{1}_{W \ominus B_{r}}\right)(x) \leq r\right\}\right)}{V_{V}(\Psi) \operatorname{vol}\left(W \ominus B_{r}\right)}
$$

is an unbiased estimator of $F_{\Xi, \Psi}(r)$ for those $r$ with $\operatorname{vol}\left(W \ominus B_{r}\right)>0$, i. e. $\operatorname{vol}\left(W \ominus B_{r}\right)$ is the window function appropriately chosen for the Euclidean distance. (If also $V_{V}(\Psi)$ is estimated from the image data then the above estimator is called ratio-unbiased.)

In our case the data are given on a point lattice, thus the EDT is applied to the image. We use the hybrid algorithm by Cuisenaire (1999) which is very efficient on large 3D images although having a worst case complexity slightly higher than linear.

Using the EDT on the lattice we measure the shortest distances of the background pixels to the foreground pixels while we are interested in the shortest distance to the complementary set. Consider two neighboring lattice points $x$ and $y$, one belonging to the foreground and the other belonging to the background. Then the boundary of $\Xi$ intersects the straight line $[x, y]$ at a point $(1-p) x+p y$ where $p \in[0,1]$. In the case of a macroscopically homogeneous set, $p$ is uniformly distributed on $[0,1]$. Its expectation is $1 / 2$ and, hence, in case of a cubic lattice half the lattice spacing must be subtracted from the distances measured by the EDT.

\section{Spatial cross-correlations in metallic foams}

In order to demonstrate the potential of the developed methods, they are now applied to investigate pore nucleation during the early foaming process of aluminium alloy foams produced by the powder-metallurgical route. We focus on 


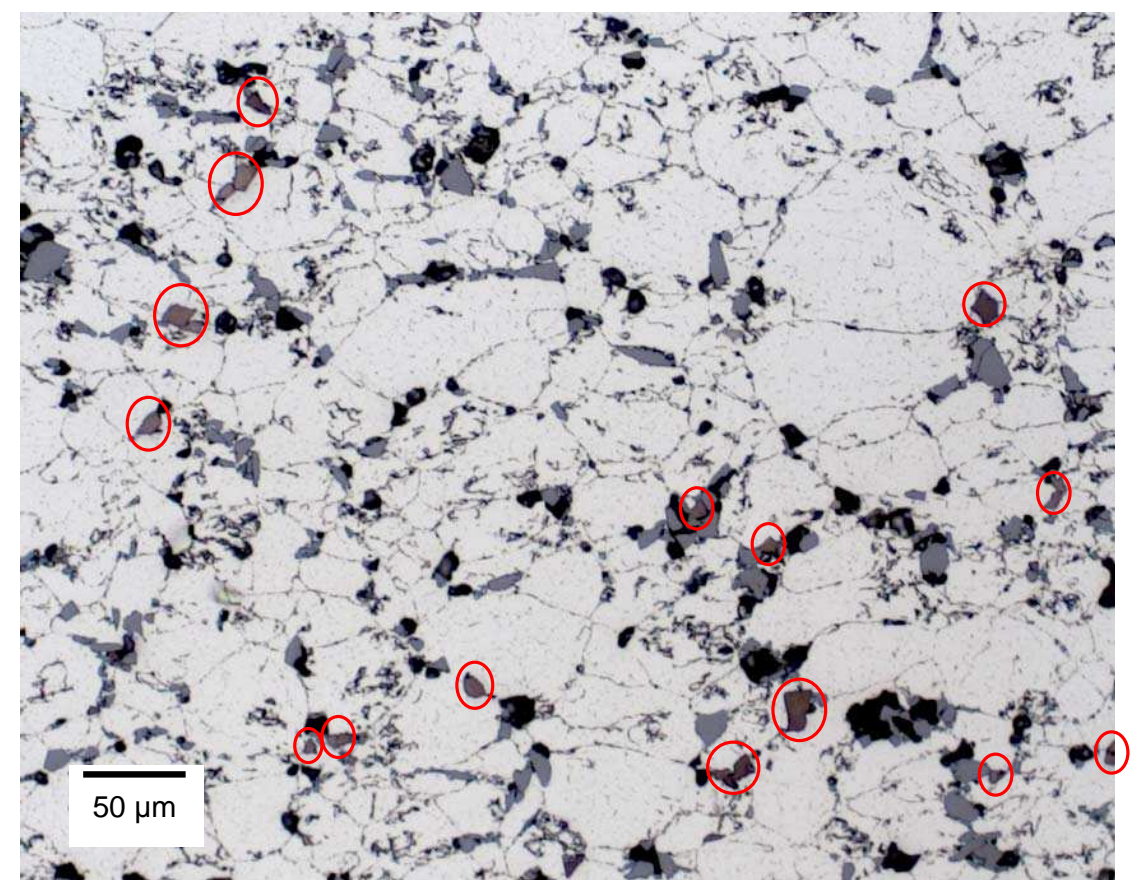

Figure 3: Metallographic image of an AlSi7 sample (1.5\% porosity, etched by $0.5 \% \mathrm{HF}$ ): aluminium particles (light grey), silicon particles (grey) and $\mathrm{TiH}_{2}$ (marked with circles) - Rack (2006).

the two possible mechanisms for pore nucleation already postulated for zinc foams (Bellmann et al., 2001) - type-I pores inflated in the direct neighborhood of blowing agent particles and type-II pores forming more distantly due to migration of the blowing gas in the foaming metal matrix, e. g. as a result of its residual porosity.

\subsection{Sample preparation}

We consider two different samples - one (called AlSi7) based on a precursor prepared by mixing elemental aluminium with the addition of elemental silicon powder and titanium hydride $\left(\mathrm{TiH}_{2}\right)$ as blowing agent, to yield a powder mix containing $7 \%$ of its weight (weight-percent - wt.- $\%$ ) silicon and 0.5 wt.- $\% \mathrm{TiH}_{2}$. For the other sample, the precursor is produced by using an aluminium alloy powder $(\mathrm{AW}-6061=\mathrm{AW}-\mathrm{AlMg} 1 \mathrm{SiCu})$ again with $0.5 \mathrm{wt} .-\% \mathrm{TiH}_{2}$ as blowing agent. The resulting two powders are compacted to dense foamable precursor materials (30 min at $12 \mathrm{MPa}$ and $450^{\circ} \mathrm{C}$, Elmoutaouakkil et al. (2002)). For both cases, the foaming is carried out by heating the precursors in a pre-heated furnace which triggers the release of hydrogen gas by the blowing agent. The heating process is soon interrupted (200 s dwell time in the furnace for AlSi7, $105 \mathrm{~s}$ for Al6061) by quenching in order to freeze the so-created early foam structures.

A very efficient first approach for the analysis of pore formation are $2 \mathrm{D}$ metallographic images of the specimens: the sample is cut, then polished along 
the resulting slice and etched in order to visualise former powder particles due to their different grain boundaries. A light microscopic image of an AlSi7 sample, as shown in Figure 3 (1.5\% porosity - early stage), shows the different elements and gives a first insight into the foaming process - in a destructive manner and without spatial (i.e. 3D) information. This metallographic image shows that most of the early pores in this AlSi7 foam are not located in the vicinity of the blowing agent particles $\mathrm{TiH}_{2}$ and therefore can be categorised as type-II (Bellmann et al., 2001). Although intuition suggests the opposite we derive as a first hypothesis that the pore formation in early AlSi7 foams is spatially independent from the position of the blowing agent particles.

\subsection{Imaging}

In order to further examine this hypothesis, synchrotron microtomography is applied to the AlSi7 and AW-6061 samples. Subsequently, correlation analysis is performed to identify the predominant location of pore nucleation. That is, the methods introduced in Sections 3.1 and 3.3 are applied to blowing agent particles and the pore structure in the resulting $3 \mathrm{D}$ images.

Specimens are cut from the produced foams, in order to fit the field of view for $\mathrm{S} \mu \mathrm{CT}$ which is closely related to the imaging resolution required. The $3 \mathrm{D}$ images are taken at the imaging beamline ID19 of the European Synchrotron Radiation Facility (ESRF), Grenoble, France - cf. Figure 1. Due to the small pores present in our samples, a pixel size of $0.7 \mu \mathrm{m}$ with a $2048 \times 2048$ pixel CCD chip is used, yielding a true spatial resolution better than $1.5 \mu \mathrm{m}$ and leading to a $1.4 \times 1.4 \mathrm{~mm}^{2}$ field of view. The maximum sample volume to be analysed with this setting would be $(1.4 \mathrm{~mm})^{3}$. The synchrotron beam is monochromatised to $17 \mathrm{keV}$ which allows easy segmentation. The resulting tomographic data sets (see Figures 4 and 5 - each $660 \times 660 \times 660$ voxels) are separated into Boolean images of the pore structure and the blowing agent according to Section 3 . Subsequently, the correlation is investigated.

\subsection{Results of image analysis}

First, we look at the AlSi7 foam in early expansion stage with a low porosity of $5.9 \%$. In Figure 6 (left) the result of the correlation analysis is plotted, showing the spatial correlation between the volume identified as pores and the volume identified as blowing agent. The values of $t_{1}$ remain close to one for all distances, indicating that there is weak cross-correlation. The distance method (Section 3.3 ) yields a similar result. For this early AlSi7 foam, the function $\delta t_{2}$ (which is roughly speaking the density of the blowing agent in dependence of its distance to the pore space normalised with the mean density of the blowing agent $\mathrm{TiH}_{2}$ in the foam matrix) is close to 1 for all distances to the pore surface indicating low stochastic dependence between blowing agent and predominantly type-II pores, see Figure 6 (right). For the sake of comparability, the quantity $t_{2}$ is presented here in a differential form. The shape of the graph of

$$
\delta t_{2}(r)=\frac{F_{\Xi, \Psi^{\prime}}(r+\delta r)-F_{\Xi, \Psi^{\prime}}(r)}{F_{\Xi, \Xi^{c}}(r+\delta r)-F_{\Xi, \Xi^{c}}(r)}, \quad r \geq 0, \delta r>0,
$$

shown in Figure 6 (right) is similar to the one of $t_{1}$, cf. Figure 6 (left). 

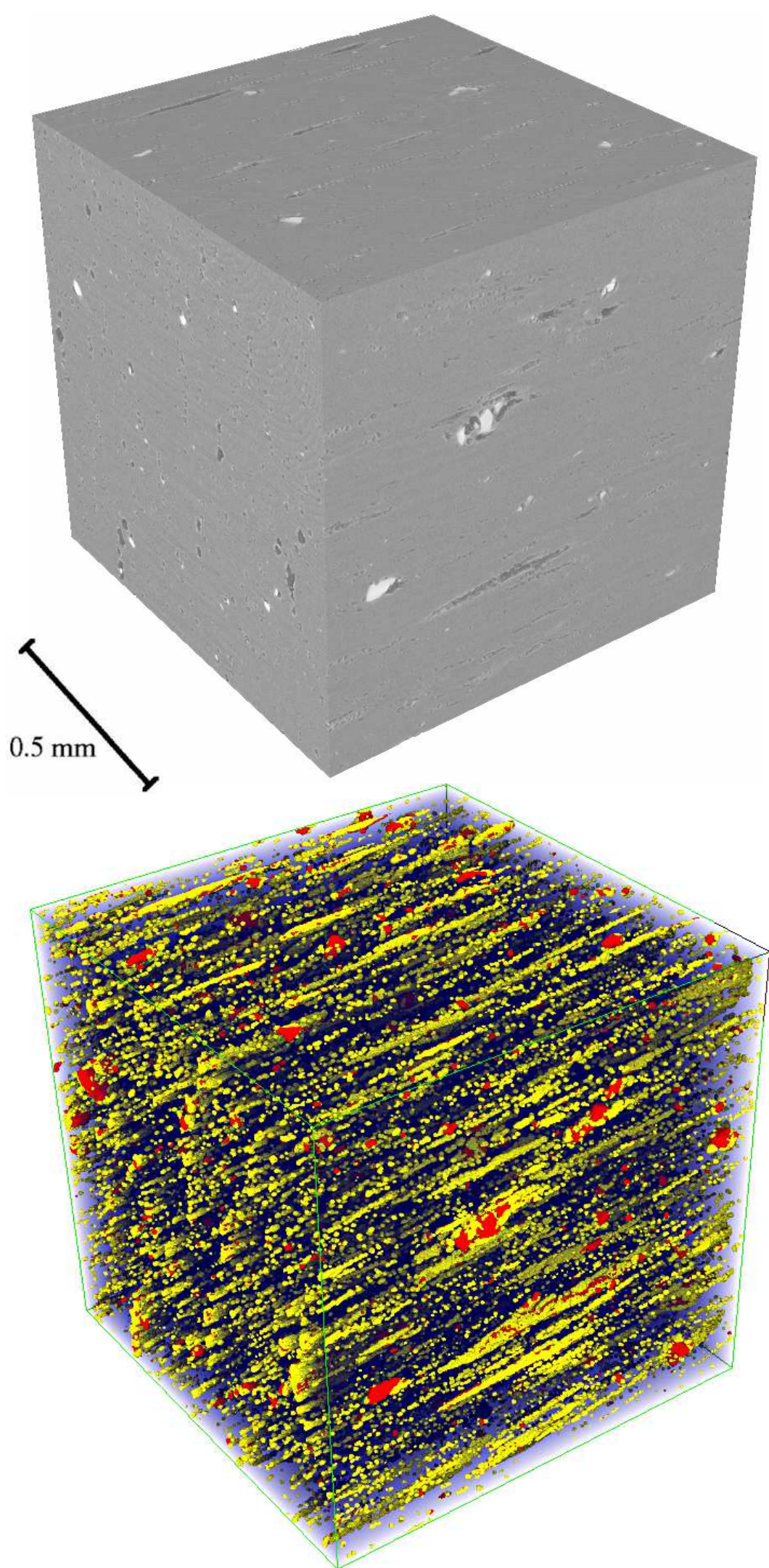
Figure 4: Top: 3D rendering of a synchrotron microtomography image $(0.7 \mu \mathrm{m}$ pixel size, $<1.5 \mu \mathrm{m}$ spatial resolution, $17 \mathrm{keV}$ X-ray photon energy) showing an AW-6061 metallic foam in early stage of expansion (2.8\% porosity): the aluminium-silicon matrix (grey), the blowing agent $\mathrm{TiH}_{2}$ (white) and the pores (black) - Helfen et al. (2003). Bottom: the same data set rendered with separated constituents (aluminium-silicon matrix in semitransparent blue, the blowing agent $\mathrm{TiH}_{2}$ in red and the pores in yellow) reveals that pores are aligned along parallel lines.

The hatched area in the plots is not accessible for analysis because of the halo effect: The image contains three constituents (pores, aluminium foam matrix, blowing agent particles). Due to the discrete sampling and the imaging system's point spread function the interface between pore and blowing agent is blurred, so that a voxel layer between pore volume and blowing agent volume contains grey-values between the pore-grey values and the blowing-agent grey values. These values are almost identical with the grey values of the aluminium foam matrix. Thus the algorithm used for creating the Boolean images identifies this layer as aluminium matrix. Therefore these voxels are omitted when applying the correlation analysis on the pore and the blowing agent volume.

The range of the abscissa in the plots is different because for the crosscorrelation method it is determined via the edge length of the investigated volume while for the distance method it is limited by the largest value in the distance map of the data set.

Going back to the metallographic image in Figure 3, one can see that most pores are located close to silicon particles or are merged with silicon particle clusters. We therefore derive the second hypothesis that in AlSi7 foams the positions of the early pores are determined by the silicon particles and not the $\mathrm{TiH}_{2}$ particles. Further investigations including holotomography on AlSi7 foams (see Helfen et al. (2005)) show spatial correlation between pores and silicon particles. Thus we derive as final conclusion that the blowing agent particles do not influence the position of the first pores in AlSi7 foams - contrary to AW-6061 foams.

Figure 7 shows the results of image analysis for the AW-6061 sample. There are larger values for $t_{1}$ at short distances to the pore space, indicating high spatial correlation between the $\mathrm{TiH}_{2}$ positions and the early pore positions. The same holds for the distance method: high values for $\delta t_{2}$ for short distances to the pore space correspond to a high density of $\mathrm{TiH}_{2}$ which indicates strong dependence between the positions of $\mathrm{TiH}_{2}$ particles and the early pores - the type-I pores.

\section{Discussion and summary}

We have introduced two new approaches to analyze spatial cross-correlation in multi-constituent volume data sets. The first one fits the common understanding of cross-correlation between constituents. It is based on the measurement of the cross-correlation function and is well-adapted to our $3 \mathrm{D}$ volume image setting where the position of one constituent never overlaps with the position of another constituent. The cross-correlation is the characteristic which is suited for our 

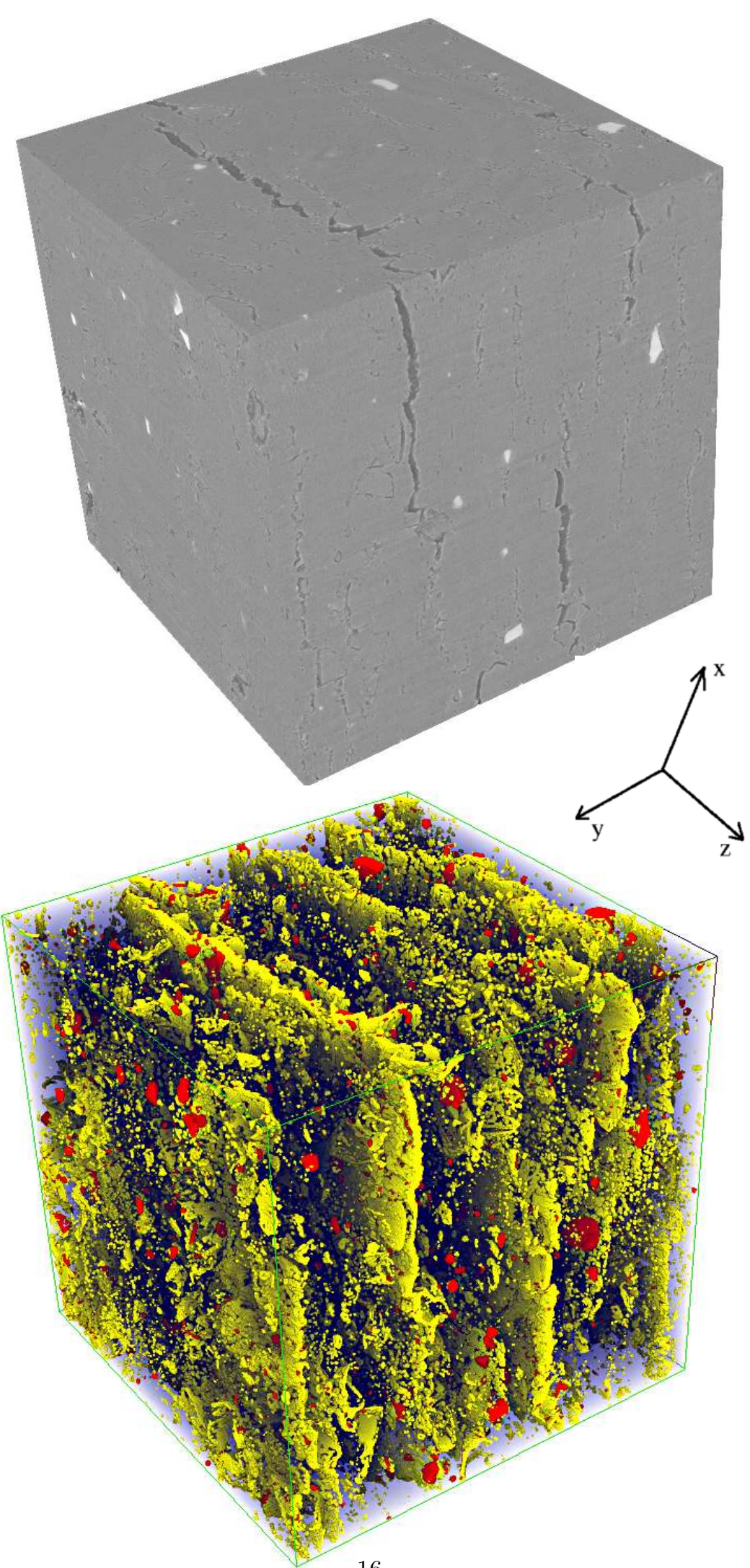
Figure 5: Top: 3D rendering of a synchrotron microtomography image $(0.7 \mu \mathrm{m}$ pixel size, $<1.5 \mu \mathrm{m}$ spatial resolution, $17 \mathrm{keV}$ X-ray photon energy) showing an AlSi7 metallic foam in an early stage of extension ( $5.9 \%$ porosity): the aluminium-silicon matrix (grey), the blowing agent $\mathrm{TiH}_{2}$ (white) and the pores (black) - Helfen et al. (2003). Bottom: the same data set rendered with separated constituents (aluminium-silicon matrix in semitransparent blue, the blowing agent $\mathrm{TiH}_{2}$ in red and the pores in yellow) reveals in contrast to AW-6061 (cf. Figure 4) a lateral extended pore structure with parallel orientation.
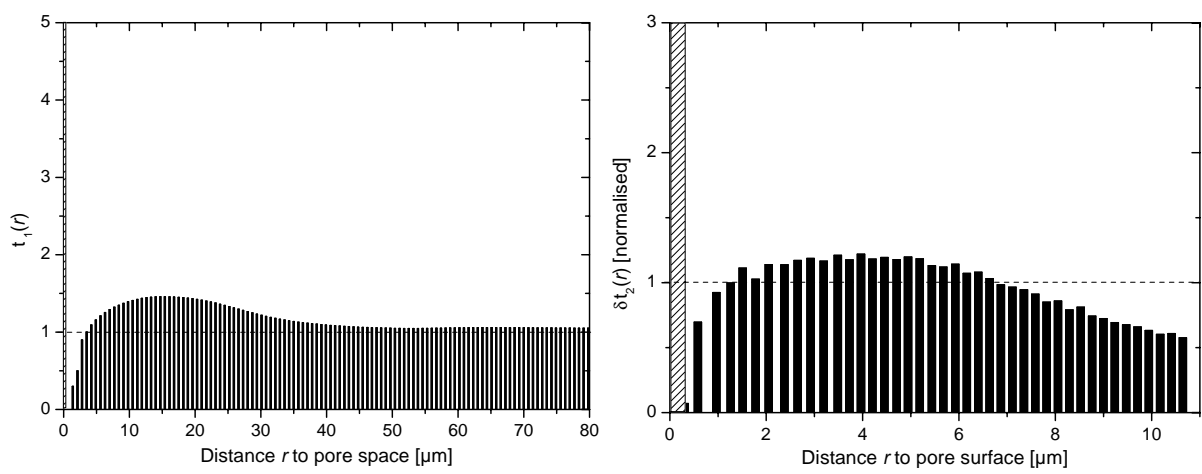

Figure 6: Spatial cross-correlation for the AlSi7 foam sample (5.9\% porosity). Left: the values of the function $t_{1}$ are close to 1 for all distances to the pore volume, indicating low cross-correlation. Right: the function $\delta t_{2}$ is close to 1 for all distances of the blowing agent to the pore surface. (Hatched areas not accessible for analyses due to halo effects.)
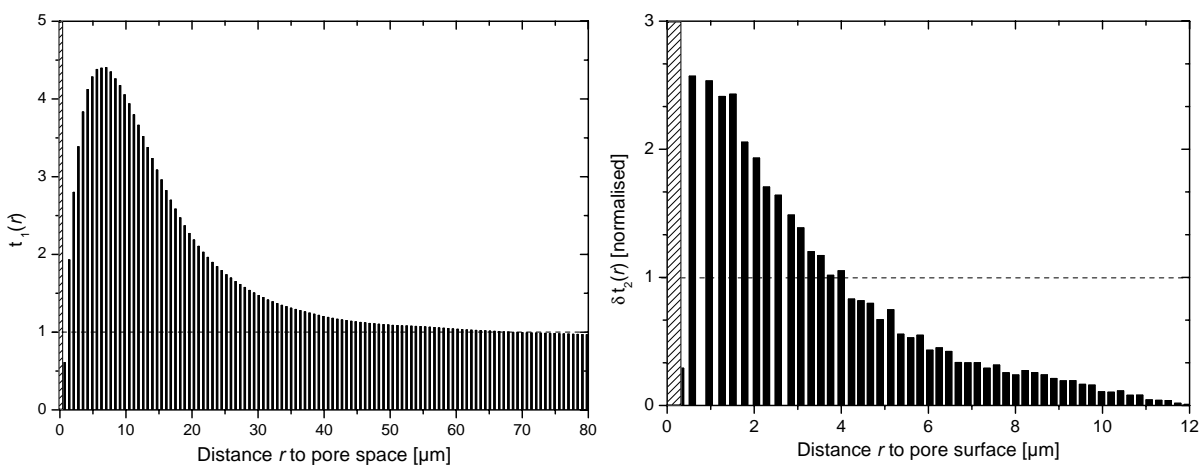

Figure 7: Spatial cross-correlation for the AW-6061 foam sample (2.8\% porosity). Left: the function $t_{1}$ showing high values for short distances to the pore space. Right: the function $\delta t_{2}$ showing high volume density of blowing agent particles close to the pore surface. (Hatched areas not accessible for analyses due to halo effects.) 
problem but its computation involves the Fourier transform with a complexity of $\mathcal{O}(n \log n)$ (where $n$ is the number of pixels) and the requirement to handle complex float values, resulting in an increased memory consumption.

Our second approach is based on determining the inter-constituent distances via the EDT, which yields a further necessary condition for stochastic independence of two constituents. The main improvement of the method compared to the algorithm described in Helfen et al. $(2003,2005)$ is the direct access to the distance information between the constituents which allows a much finer discretisation of the abscissa range in the plots 6 (right) and 7 (right). Compared to the Fourier-based method, processing times are shorter as well as memory consumption is four times lower as fast algorithms for the EDT are only of complexity $\mathcal{O}(n)$ and require to handle real float values only. Memory consumption is particularly important as the size of microtomography data sets can go up to several tens of gigabytes and the memory available even in modern 64bit machines is still limited compared to that.

The distance method delivers a quantity describing the shortest distance of the volume elements of one constituent (in our example the blowing agent) to the surface of another (here the pore space). In our example application of this quantity could be found easier to interpret than the cross-correlation of the volume elements assigned to different constituents. Nevertheless, this is a matter of taste.

We can summarize that our investigations of AW-6061 and AlSi7 foams show that two types of pores exist in the early stages of the foaming process. For the AW-6061 sample made from a rather homogeneous precursor consisting only of AW-6061 and the blowing agent $\mathrm{TiH}_{2}$ we find type-I pores which are spatially correlated with the positions of the $\mathrm{TiH}_{2}$ particles. The AlSi7 sample with its heterogeneous precursor consisting of aluminium and silicon as elementary materials and $\mathrm{TiH}_{2}$, shows no spatial correlation between pores and blowing agent particles' positions, indicating predominantly type-II pores. This suggest that the way of manufacturing the material to be foamed strongly influences the resulting pore structure. The materials science aspect of our results will be discussed more detailed in a forthcoming publication.

\section{Acknowledgments}

Katja Schladitz was supported by the Rheinland-Pfalz cluster of excellence "Dependable Adaptive Systems and Mathematical Modeling" (www.dasmod.de).

The research of J. Ohser was supported by the $\mathrm{FH}^{3}$-programme of the German Federal Ministry of Education and Research under project grant 1711B06.

We thank Rebekka Malten for image and data processing, Heiko Stanzick for sample preparations, Tetyana Sych for the beautiful volume renderings, the reviewers for the useful remarks and Petra Pernot for experimental support.

The image analysis was performed using Fraunhofer ITWM's MAVI software Fraunhofer ITWM, Department of Image Processing (2005). 


\section{References}

Agterberg, F. P. \& Fabbri, A. G. (1979). Spatial correlation of stratigraphic units quantified from geological maps. Computers \& Geosci. 4, 515-526.

Banhart, J. (2001). Manufacture, characterisation and application of cellular metals and metal foams. Prog. Mat. Sci. 46, 559-632.

Banhart, J., ed. (2007). Advanced Tomographic Methods in Materials Research and Engineering. Oxford University Press, Oxford.

Banhart, J. \& Weaire, D. (2002). On the road again - metal foams find favour. Physics Today 55, 37-42.

Bellmann, D., Banhart, J. \& Clemens, H. (2001). Investigation of metal foam formation by microscopy and ultra small-angle neutron scattering. Acta $M a-$ terialia 49, 3409-3420.

Bonse, U. \& Busch, F. (1996). X-ray computed microtomography ( $\mu$ CT) using synchrotron radiation (SR). Prog. Biophys. Molec. Biol. 65, 133-169.

Cloetens, P., Ludwig, W., Baruchel, J., Van Dyck, D., Van Landuyt, J., Guigay, J. P. \& Schlenker, M. (1999). Holotomography: Quantitative phase tomography with micrometer resolution using hard synchrotron radiation X-rays. Appl. Phys. Lett. 75, 2912-2914.

Cuisenaire, O. (1999). Distance transformations: fast algorithms and applications to medical image processing. Ph.D. thesis, Université catholique de Louvain, http://www.tele.ucl.ac.be/PEOPLE/OC/these.pdf.

Duffy, G. P. \& Hughes-Clarke, J. E. (2005). Application of spatial cross correlation to detection of migration of submarine sand dunes: Marine sandware and river dune dynamics. J. Geophys. Res. 110, 1-11.

Elmoutaouakkil, A., Salvo, L., Maire, E. \& Peix, G. (2002). 2D and 3D characterization of metal foams using x-ray tomography. Adv. Eng. Mat. 4, 803-807.

Flannery, P. B., Deckmann, H. W., Roberge, W. G. \& D'Amico, K. L. (1987). Three-dimensional X-ray microtomography. Science 237, 1439-1444.

Frank, J. (1980). The role of correlation techniques in computer image processing. In: Computer Processing of Electron Microscope Images (ed. P. Hawkes), Springer, Berlin, 187-222.

Fraunhofer ITWM, Department of Image Processing (2005). MAVI - modular algorithms for volume images. http://www.itwm.fhg.de/mab/projects/ MAVI/.

Helfen, L., Stanzick, H., Ohser, J., Schladitz, K., Pernot, P., Banhart, J. \& Baumbach, T. (2003). Investigation of the foaming process of metals by synchrotron-radiation imaging. In: Proceedings SPIE: Testing, Reliability, and Application of Micro- and Nanomaterial Systems (eds. N. Meyendorf, G. Baaklini \& B. Michel), volume 5045, 254-265. 
Helfen, L., Baumbach, T., Pernot, P., Cloetens, P., Stanzick, H., Schladitz, K. \& Banhart, J. (2005). Investigation of pore initiation in metal foams by synchrotron-radiation tomography. Appl. Phys. Lett. 86, 231907-1-231907-3.

Hsieh, J. (2003). Computed Tomography - Principles, Design, Artifacts and Recent Advances. SPIE Press.

Jeulin, D. (1986). Study of spatial distributions in multicomponent structures by image analysis. Acta Stereol. 5/2, 233-239.

Jorswieck, E. A. \& Sezgin, A. (2004). Impact of spatial correlation on the performance of orthogonal space-time block codes. IEEE Communications Letters 8, 21-23.

Koch, K., Ohser, J. \& Schladitz, K. (2003). Spectral theory for random closed sets and estimating the covariance via frequency space. Adv. Appl. Prob. 35, 603-613.

Lhotský, J. (2006). Density of cross-correlation measure and stationarity: overview. In: Proceedings of the International Conference on Stereology, Spatial Statistics and Stochastic Geometry, Prague, June 26-29 (eds. R. Lechnerová, I. Saxl \& V. Beneš), Union of Czech Methematicans and Physicists, Prague, 97-102.

Ma, J., Zeng, D. \& Chen, H. (2006). Spatial-temporal cross-correlation analysis: A new measure and a case study in infectious disease informatics. In: Intelligence and Security Informatics, IEEE International Conference on Intelligence and Security Informatics, ISI 2006, San Diego, CA, USA, May 23-24 (eds. S. Mehrotra, D. D. Zeng, H. Chen, B. M. Thuraisingham \& F.-Y. Wang), volume 3975 of Lecture Notes in Computer Science, Springer, Berlin, Heidelberg, 542-547.

Manolakis, D. \& Proakis, J. (1996). Digital Signal Processing. Prentice-Hall, 3rd edition.

Marcotte, D. (1996). Fast variogram computation with FFT. Computers \& Geosci. 22, 1175-1186.

Mayer, J. (2004). A time-optimal algorithm for the estimation of contact distribution functions of random sets. Image Anal. Stereol. 23, 177-183.

Ohser, J. \& Mücklich, F. (2000). Statistical Analysis of Microstructures in Materials Science. J Wiley \& Sons, Chichester, New York.

Ohser, J., Schladitz, K., Koch, K. \& Nöthe, M. (2005). Diffraction by image processing and its application in materials science. Z. Metallkunde 96, 731737 .

Pal, N. R. \& Bhandari, D. (1993). Image thresholding: Some new techniques. Signal Processing 33, 139-158.

Pal, N. R. \& Pal, S. K. (1993). Review on image segmentation techniques. Pattern Recognition 26, 1277-1294. 
Rack, A. (2006). Charakterisierung komplexer Materialsysteme mittels Synchrotron-Tomographie und 3D-Bildanalyse. Ph.D. thesis, Technical University Berlin, http://opus.kobv.de/tuberlin/volltexte/2006/1370/.

Reich, R. M., Czapalewski, R. L. \& Bechtold, W. A. (1994). Spatial crosscorrelation of undisturbed, natural shortleaf pine stands in northern Georgia. Environmental Ecol. Stat. 1, 201-217.

Reimers, P. \& Goebbels, J. (1983). New possibilities of nondestructive evaluation by x-ray computed tomography. Mat. Eval. 41, 732-737.

Stoyan, D. (1984a). Correlations of the marks of marked point processes - statistical inference and simple models. J. Inf. Process. Cybern. 20, 285-294.

Stoyan, D. (1984b). On correlations of marked point processes. Math. Nachr. 116, 197-207.

Stoyan, D. \& Ohser, J. (1982). Correlations between planar random structures, with an ecological application. Biom. J. 24, 631-647.

Stoyan, D. \& Ohser, J. (1984). Cross-correlation measure of weighted random measures and their estimation. Teor. Verojatn. Primen. 29, 338-347.

Torquato, S. (2002). Random Heterogeneous Materials: Microstructure and Macroscopic Properties. Springer, New York.

Wirjadi, O. (2007). Survey of 3d image segmentation methods. Technical Report 123, Fraunhofer ITWM, Kaiserslautern.

Yu, C. J., Eifert, H., Banhart, J. \& Baumeister, J. (1998). Metal foaming by a powder metallurgical method: production, properties and applications. Mat. Res. Innovat. 2, 181-188. 